\title{
SIMULAÇÃO DA PERFORMANCE DA EVAPORAÇÃO DE MÚLTIPLO EFEITO DE LICOR POBRE OPERANDO CONTINUAMENTE
}

\author{
F. A. PONTES ${ }^{1}$, E. T. CABRAL ${ }^{1}$, J. N. N. QUARESMA ${ }^{2}$, C. da S. BATISTA ${ }^{2}$ e A. C. de \\ CARVALHO ${ }^{3}$ \\ ${ }^{1}$ Universidade Federal do Pará, Programa de Pós Graduação em Engenharia Química \\ ${ }^{2}$ Universidade Federal do Pará, Faculdade de Engenharia Química \\ ${ }^{3}$ Universidade Federal do Pará, Mestrado Profissional em Engenharia de Processos \\ E-mail para contato: fabioapbelem@hotmail.com
}

\begin{abstract}
RESUMO - O presente trabalho utilizou-se do software Excel para estimar o comportamento do sistema de evaporação de licor pobre operando continuamente com dez efeitos em uma refinaria de alumina. A simulação do comportamento desta área é importante uma vez que existe a necessidade de se otimizar o controle de diversos parâmetros de processo da evaporação, a qual pode ser feita a partir da modelagem e simulação do comportamento do processo da área, influenciando de forma positiva no controle de volume da planta, bem como resultando em melhorias operacionais, de processo, de segurança, de meio ambiente, e até mesmo na questão da limpeza e manutenção dos equipamentos. O desenvolvimento dos modelos da evaporação foi realizado através de balanço de massa global e parcial bem como balanço de energia em estado estacionário, equação de flash, equação de Antoine e interpolações para entalpia do líquido e do vapor, para cada unidade de processamento. Os resultados obtidos foram comparados com os dados de projeto fornecidos pela empresa a fim de verificar a validade do simulador observando-se uma aproximação bastante aceitável para todos os parâmetros estudados.
\end{abstract}

\section{INTRODUÇÃO}

O processo Bayer consiste na dissolução da gibbsita presente na bauxita em uma solução de licor caustico, em moderada temperatura e pressão no interior de um digestor, resultando em uma polpa cuja fase líquida possui elevado teor de aluminato de sódio, e que após separação da lama vermelha por meio de decantação, o hidrato de alumínio é cristalizado em tanques precipitadores com auxílio de resfriamento do licor Bayer e adição de sementes de hidrato.

Em seguida o hidrato precipitado é separado em classificadores gravimétricos que particionam a fase sólida da precipitação em hidrato-produto e sementes, bem como separa a fase líquida como licor pobre (pobre em aluminato de sódio) em espessadores de hidrato, que deve retornar a área da digestão passando antes pela área de resfriamento à vácuo, onde o mesmo é aquecido em trocadores de calor 


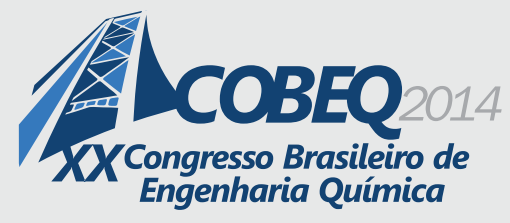

do tipo casco e tubo utilizando o vapor regenerativo vindo da expansão do licor rico (rico em aluminato) que é resfriado antes de entrar na área de precipitação.

Após este aquecimento, o licor pobre deve ser direcionado à área de evaporação de licor pobre (ou simplesmente evaporação), etapa na qual o licor é concentrado em flash tanks (FTs) a partir da expansão da água (cosolvente) em FTs e trocadores de calor do tipo casco e tubo (HEs do inglês heat exchangers). A seguir é mostrado o fluxograma da área de evaporação na Figura 1:

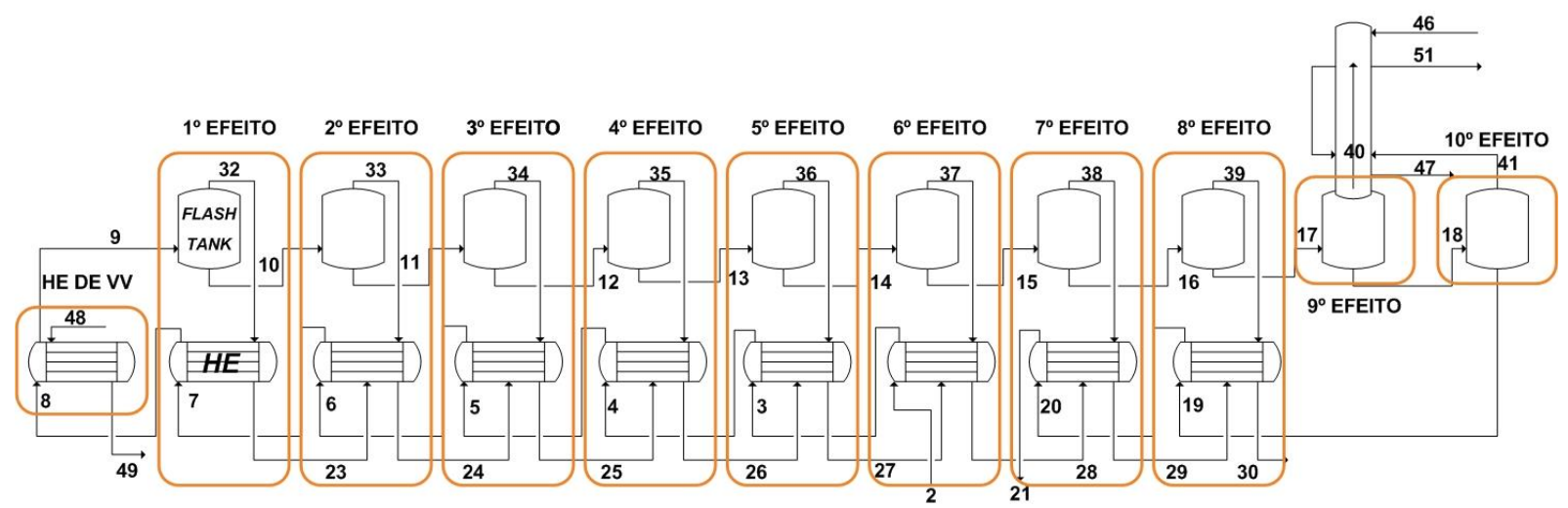

Figura 1 - Fluxograma do processo de evaporação de licor pobre com detalhe nos efeitos.

A justificativa da realização deste trabalho encontra-se na necessidade de se otimizar o controle de diversos parâmetros de processo da evaporação, otimização essa que pode ser alcançada a partir da modelagem e simulação do comportamento do processo, principalmente em cenários adversos, de forma que com o auxílio de um simulador, como uma ferramenta de análise, podem ser obtidas diversas melhorias operacionais, de processo, de segurança, de meio ambiente, e até mesmo na questão da limpeza e manutenção dos equipamentos, dentre as quais destacam-se:

Estabilidade operacional: o uso do simulador pode proporcionar uma maior previsibilidade da condição ótima de operação da área, resultando em um melhor controle do processo de evaporação, sendo possível atuar sobre anomalias e falhas de operação mais eficientemente.

Custo de conversão: um processo de evaporação controlado tem como consequência um melhor controle sobre o volume da planta, o que gera uma menor contribuição do mesmo nos custos de conversão, de forma a proporcionar um maior ganho energético pela redução de gastos com vapor vivo, limpeza ácida e cáustica, manutenção de equipamentos, reagentes, tratamento de efluentes, dentre outros.

Qualidade do produto: um processo controlado resulta em parâmetros de controle na meta, resultando em maior controle sobre a qualidade do produto.

O objetivo deste trabalho consiste no desenvolvimento de um simulador via software Excel, do comportamento da área de evaporação de licor pobre em múltiplos efeitos que opera continuamente em uma refinaria de alumina, baseado em modelos de balanço de massa e de energia em estado estacionário, de modo a fornecer uma ferramenta de controle de processo. 


\section{METODOLOGIA DE SOLUÇÃO}

A modelagem do problema em questão foi realizada com base na lei da conservação da massa e na primeira lei da Termodinâmica; na equação de Antoine para o modelo da temperatura do vapor a partir da pressão de vapor e nas interpolações dos dados da tabela de vapor para modelar as entalpias de líquidos e de vapor da água pura. A demonstração dos balanços é feita para o primeiro estágio a seguir. Na Figura 2 observa-se o esquema do primeiro estágio da evaporação:

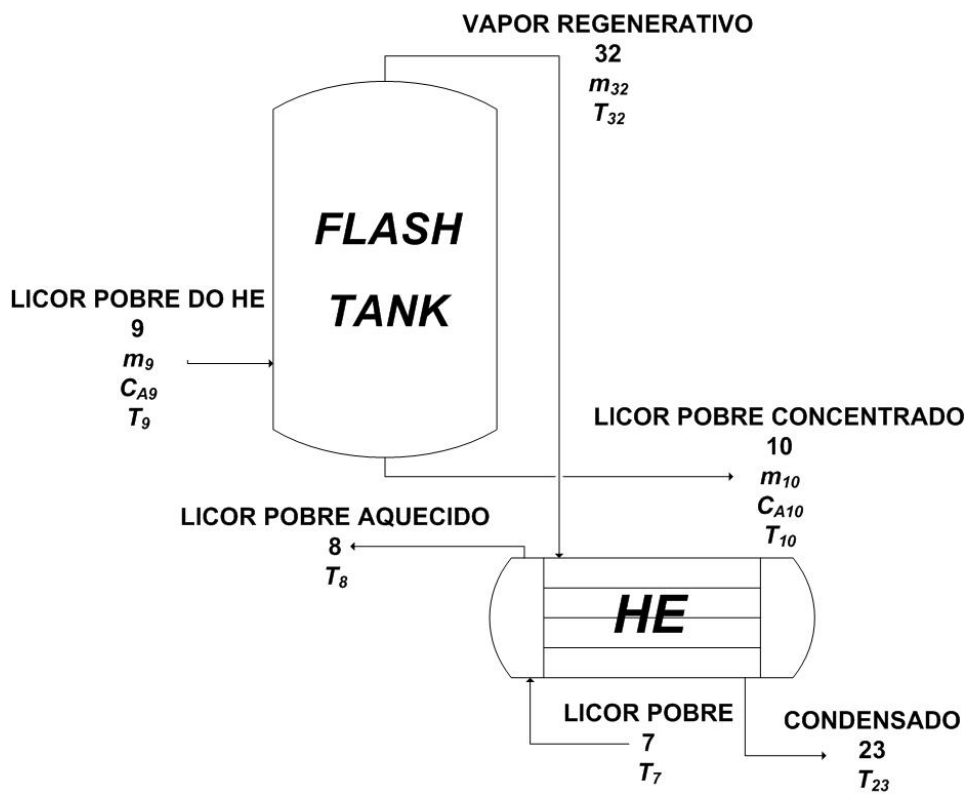

Figura 2 - Primeiro efeito do processo de evaporação de licor pobre.

\subsection{Balanço de massa global e parcial no FT}

Os balanços de massa global e parcial no flash tank resultam nas Equações (1) e (2), respectivamente, mostradas a seguir (FELDER e ROUSSEAU, 2005):

$$
\begin{aligned}
& m_{10}=m_{9}-m_{32} \\
& C_{A, 10}=\frac{m_{9} C_{A, 9}}{m_{10}}
\end{aligned}
$$

\subsection{Modelo de retirada de vapor}

O cálculo da retirada de vapor é feito pela equação de vapor de flash mostrada abaixo (SPIRAX SARCO, 2013): 


$$
m_{32}=m_{9}\left(\frac{H_{9}^{L}-H_{10}^{L}}{H_{L, 100^{\circ} \mathrm{C}}^{V}}\right)
$$

\subsection{Modelo para entalpia do líquido}

A interpolação para a entalpia do líquido (fase líquida do licor) de entrada e de saída do tanque, $H_{i}^{L}$, em função da temperatura resultou no modelo a seguir (SHAPIRO, 2010):

$$
H_{i}^{L}\left(T_{i}\right)=4,2161 T_{i}-2,1018
$$

Com coeficiente de correlação:

$$
R^{2}=0,9999
$$

\subsection{Modelo para entalpia do vapor}

A interpolação para a entalpia do vapor de flash do tanque de expansão, $H_{i}^{V}$, em função da pressão de vapor resultou no seguinte modelo (SHAPIRO, 2010):

$$
H_{i}^{V}\left(P_{i}\right)=-2,5342 P_{i}+2510,3
$$

Com coeficiente de correlação:

$$
R^{2}=0,9994
$$

\subsection{Balanço de energia no HE}

Para o cálculo da temperatura do licor pobre que deixa o trocador é aplicado o seguinte balanço de energia (FELDER e ROUSSEAU, 2005):

$$
T_{8}=\frac{m_{32} \cdot H_{32}-m_{23} \cdot H_{23}}{m_{L P} \cdot c_{L P}}+T_{7}
$$

Os cálculos para os demais estágios são bastante semelhantes mudando apenas em alguns detalhes nos estágios finais onde não há acoplamento entre FTs e HEs, nem recuperação de energia.

\section{RESULTADOS E DISCUSSÃO}

O simulador do comportamento da área de evaporação de licor pobre foi desenvolvido adicionando as equações: do balanço de massa global para calcular as vazões de licor pobre, do balanço de massa parcial para calcular as concentrações cáusticas de licor pobre, de retirada de vapor 
regenerativo, da temperatura do vapor regenerativo e do balanço de energia nos HEs para calcular a temperatura do licor pobre nos HEs, no simulador desenvolvido no software Excel.

Desse modo, com um input de uma base mássica de licor pobre de $m_{2}=100 \mathrm{~m}^{3} / \mathrm{h}$, concentração mássica de cáustico: $C_{A, 2}=44,836 \mathrm{~g} / \mathrm{L}$ e temperatura do licor de entrada de $T_{2}=$ $79^{\circ} \mathrm{C}$, foi possível obter perfis dos seguintes parâmetros: vazão e concentração de licor pobre nos FTs, vazão de retirada e temperatura do vapor regenerativo dos FTs e temperatura de licor pobre nos HEs, nos efeitos de evaporação de 1 até 10 (correntes de $m_{9}$ até $m_{19}$ para o circuito de licor nos FTs, correntes de $m_{32}$ até $m_{41}$ para o circuito de vapor e correntes de $m_{2}$ até $m_{9}$ para o circuito de licor nos HEs), a fim de comparar os mesmos com os dados de projeto e verificar a validade do simulador. A seguir são mostrados os resultados e as devidas comparações dos perfis dos parâmetros avaliados neste trabalho.

Comparando os dados de projeto para a vazão de licor pobre no circuito dos FTs com o perfil resultante do simulador para a mesma vazão, conforme mostra a Figura 3, é possível afirmar que o perfil resultante da simulação não apresenta qualquer incoerência, uma vez que exibe comportamento decrescente, resultado da diminuição na vazão de licor pela retirada de vapor em cada FT. O resultado da simulação pode ser considerado excelente, uma vez que se observa que os dois perfis apresentam-se bastante próximos graficamente. Deste modo, o simulador da vazão de licor pobre nos FTs resultou em valores satisfatórios, sendo o mesmo validado.

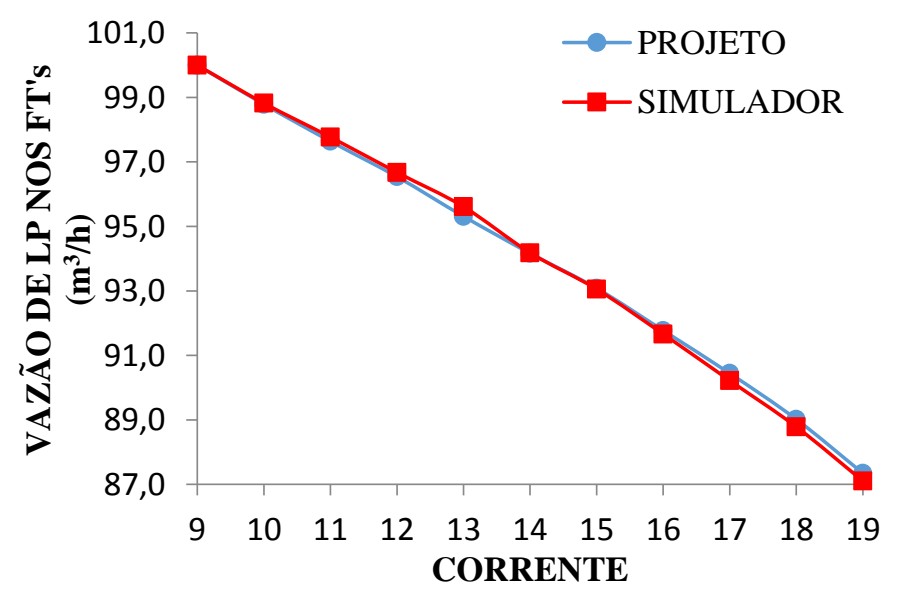

Figura 3 - Perfil de vazão de licor pobre no circuito de FTs.

Observando-se os dados de projeto juntamente com os perfis de concentração cáustica de licor pobre no circuito dos FTs do simulador, conforme ilustra a Figura 4, é possível dizer que os mesmos estão coerentes com o que se esperava, já que o perfil apresentou comportamento crescente (aumento da concentração cáustica pela retirada de água da solução), e que, pela comparação da curva da concentração simulada com a de projeto, é possível afirmar que o resultado para este parâmetro foi coerente e o simulador pode ser validado para o mesmo. 


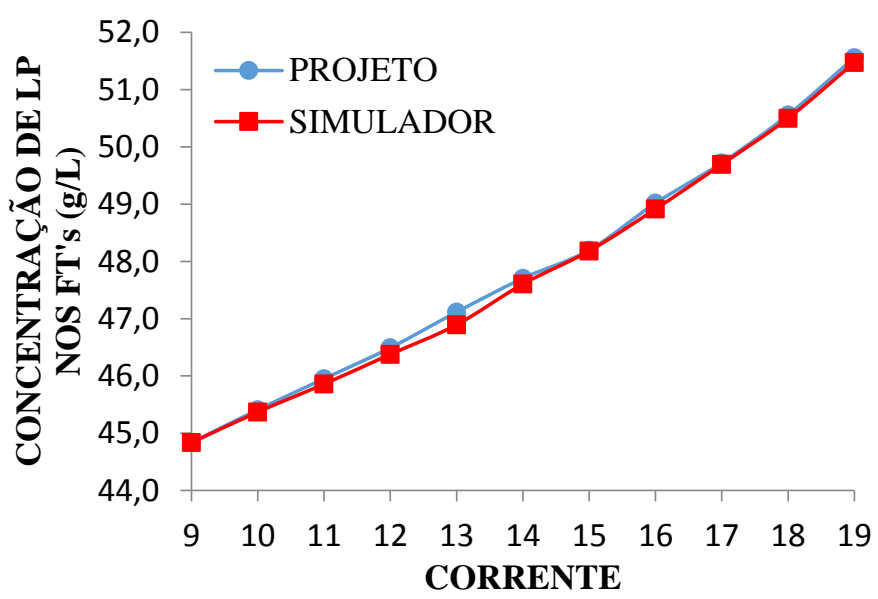

Figura 4 - Perfil de concentração cáustica de licor pobre no circuito de FTs.

Quanto ao parâmetro retirada de vapor regenerativo dos FTs, mostrado na Figura 5, é possível visualizar que, pontualmente (em cada tanque flash), há uma pequena discordância entre os valores da retirada de vapor calculado e o de projeto. No entanto, quando considera-se uma retirada total de vapor regenerativo, ou seja, a soma das vazões de vapor retirado de todos os FTs $(78,6 t / h)$, nota-se que há uma aproximação mais aceitável deste valor com o de projeto $(77,2 \mathrm{t} / \mathrm{h})$, ressaltando-se também que este modelo obedece o balanço de massa global de toda a área de evaporação.

Assim, como o vapor regenerativo segue no mesmo circuito de condensado após aquecer o licor nos HEs, essa pequena diferença pontual pode não representar nenhum problema na modelagem e simulação deste parâmetro, resultando que o modelo da retirada foi aceito e o simulador foi validado para a retirada de vapor.

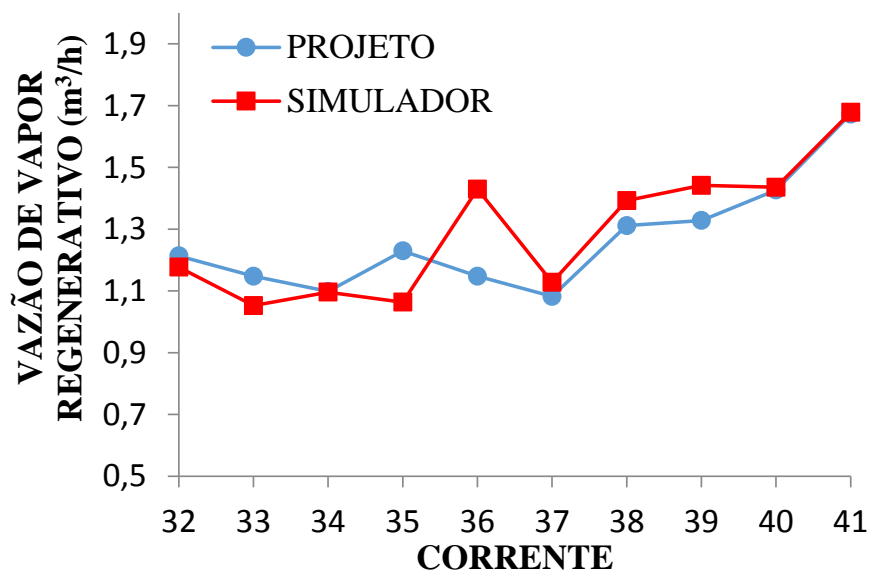

Figura 5 - Perfil de retirada de vapor dos FTs.

Em se tratando da temperatura do vapor regenerativo, de acordo com a Figura 6, pode-se afirmar que o comportamento decrescente da temperatura do vapor (resultado do resfriamento do 
licor de um FT para outro) foi bastante aceitável, acompanhando a curva dos dados de projeto. Houve uma coincidência maior dos valores calculados com aqueles de projeto obtidos na empresa quando comparado com os demais parâmetros. Assim, a verificação do simulador para este parâmetro foi alcançada satisfatoriamente.

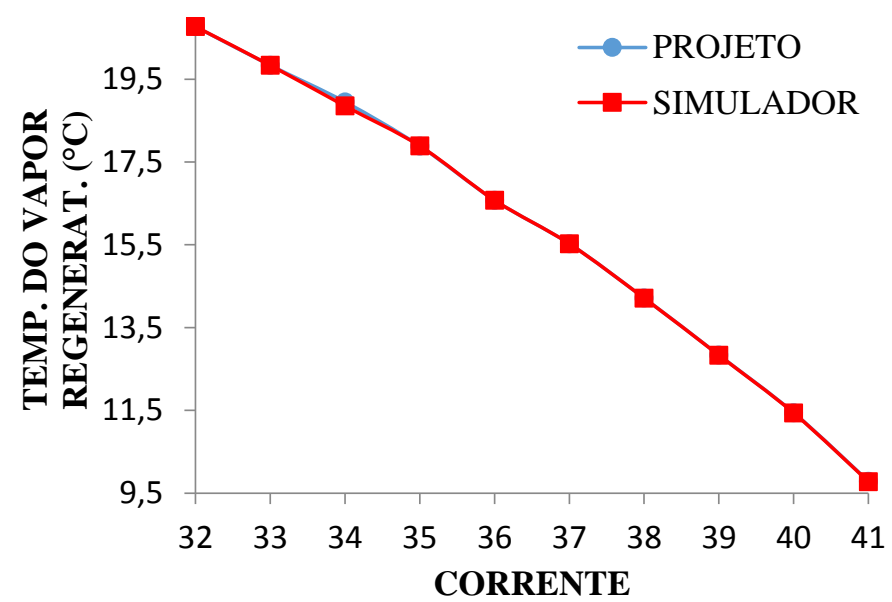

Figura 6 - Perfil de temperatura do vapor no circuito de FTs.

Os resultados da temperatura do licor pobre nos HEs pelo simulador foram considerados bastante próximos aos dados de projeto, como se observa pelos perfis de temperatura ilustrados na Figura 7. O perfil crescente da temperatura neste circuito foi considerado coerente, uma vez que era esperado este comportamento, como função do aquecimento do licor, sendo possível afirmar que este modelo foi validado e o simulador pode ser aplicado neste parâmetro.

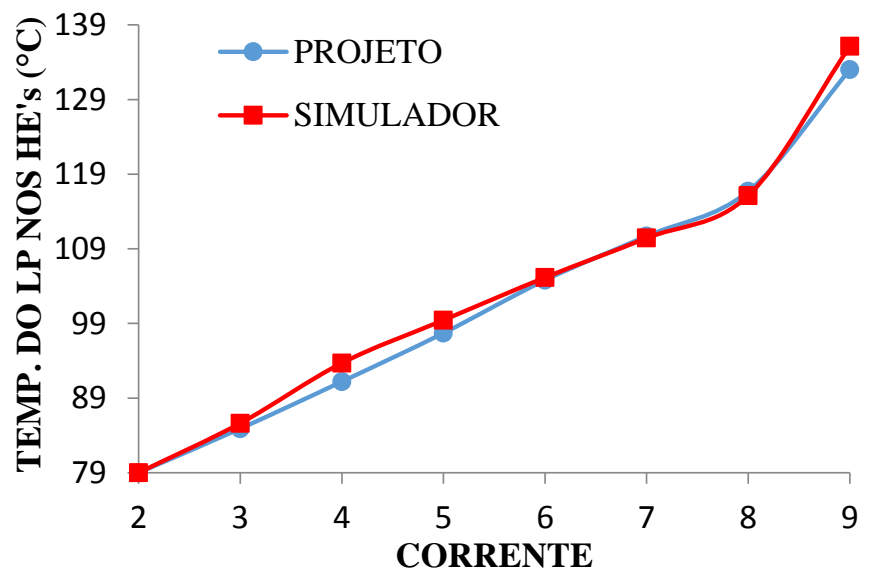

Figura 7 - Perfil de temperatura do licor pobre no circuito de HEs.

A partir da observação dos resultados ilustrados pelos gráficos mostrados, nota-se que o comportamento de todos os parâmetros foi coerente, ou seja, de acordo com o que se esperava do 
fenômeno de evaporação, de modo que, o resultado da verificação da validade do simulador foi considerado positivo.

\section{CONCLUSÃO}

A partir dos resultados apresentados e da análise feita sobre os mesmos foi possível concluir que:

Para o parâmetro vazão de licor pobre no circuito dos FTs o simulador do mesmo foi validado, uma vez que houve uma aproximação das curvas simulada e de projeto, bem como foi observado que o perfil de vazão resultante do balanço foi bastante coerente com o que se esperava.

Para a concentração cáustica de licor pobre nos FTs foi obtida uma curva bastante próxima ao projeto, confirmando a validade do modelo na solução dos problemas da evaporação.

Para a retirada de vapor regenerativo dos FTs observou-se que por mais que os valores pontuais tenham sido diferentes dos dados de projeto, a retirada total de vapor foi bem próxima do valor projetado, de modo a validar a utilização do simulador.

Quanto à temperatura do vapor regenerativo dos FTs houve um comportamento bastante satisfatório para o perfil simulado, bem como observou-se que os perfis (simulador e de projeto) foram coincidentes, o que confirmou a verificação do simulador.

Para a temperatura do licor pobre no circuito dos HEs os resultados do simulador convergiram com os de projeto, de modo a validar o modelo do comportamento da área da evaporação.

Conclui-se, deste modo que o objetivo deste trabalho foi alcançado, uma vez que os dados foram simulados de forma satisfatória e, consequentemente, o simulador foi bem validado, podendo ser utilizado na simulação do comportamento da área de evaporação.

\section{REFERÊNCIAS BIBLIOGRÁFICAS}

SHAPIRO H., MORAN M., Princípios da Termodinâmica para Engenharia. 6 ed. LTC, 2010.

SPIRAX SARCO, Flash Steam, On line. Disponível em: <http://www.spiraxsarco.com/resources/steam-engineering-tutorials/condensate-recovery/flashsteam.asp>. Acesso em: 25 jan. 2013.

FELDER R. M., ROUSSEAU R. W., Princípios Elementares dos Processos Químicos. $3^{\text {a }}$ edição. Rio de Janeiro. Editora LTC. 2005. 